\title{
ALIMENTAÇÃO DAS ESPÉCIES DE SIPHLOPHIS FITZINGER (SERPENTES, COLUBRIDAE, XENODONTINAE, PSEUDOBOINI) ${ }^{1}$
}

\author{
Ana Lúcia da Costa Prudente 2,3 \\ Julio Cesar de Moura-Leite 2,3 \\ Sérgio Augusto Abrahão Morato ${ }^{3,4}$
}

\begin{abstract}
Diet of species of Siphlophis Fitzinger (Serpentes, Colubridae, Xenodontinae, Pseudoboini). Survey of 30 stomach and/or gut contents of the five species of Siphlophis Fitzinger, 1843 revealed predominance of mostly diurnal species of lizards. Remains of nocturnal prey and snakes were also found. For the first time, one frog and one bat are recorded as preys of one species (S. cervinus Laurenti, 1768). Predation on mollusks and birds is not confirmed herein.

KEY WORDS. Serpentes, Colubridae, Xenodontinae, Pseudoboini, Siphlophis, diet
\end{abstract}

O gênero Siphlophis Fitzinger, 1843 compreende um grupo de colubrídeos neotropicais pertencentes à tribo Pseudoboini. Foram registrados, para estas serpentes, hábitos noturnos, terrestres e semiarborícolas (DUELLMAN 1990; ZIMMERMAN \& RoDRIGUES 1990; SAZIMA \& ARGÔLO 1994).

Espécies de Siphlophis foram reportadas predando aves (CUNHA \& NASCIMENTO 1978; 1993), lagartos (CUNHA \& NASCIMENTO 1978, 1993; DUELLMAN 1978; NASCIMENTO et al. 1987; SAZIMA \& ARGÔLO 1994), ovos de lagartos, serpentes (SAZIMA \& ARGÔLO 1994) e moluscos (AMARAL 1927, 1933). Entretanto, parte desses registros não são fatuais e provêm de extrapolações a partir de analogias com outras espécies de hábitos presumivelmente similares e morfologia semelhante [AMARAL (1933) referindo-se a "dormideiras" em geral].

A partir da análise de itens alimentares encontrados no trato digestivo de exemplares de Siphlophis, efetuou-se uma comparação com os dados disponíveis na literatura e foram traçadas algumas considerações sobre a dieta das espécies. Dados sobre S. longicaudatus (Andersson, 1901), S. leucocephalus (Günther, 1863) e $S$. worontzowi (Prado, 1940) são apresentados pela primeira vez.

1) Contribuição número 1059 do Departamento de Zoologia, Universidade Federal do Paraná.

2) Pós-Graduação em Ciências Biológicas Área Zoologia, Departamento de Zoologia, Universidade Federal do Paraná. Caixa Postal 19020, 81531-990 Curitiba, Paraná, Brasil. Bolsista do CNPq.

3) Laboratório de Herpetologia, Museu de História Natural Capão da Imbuia, Departamento de Zoológico, Prefeitura Municipal de Curitiba. Rua Prof. Benedito Conceição 407. 82810-080, Curitiba, Paraná, Brasil.

4) Coordenadoria de Impactos Ambientais, Companhia Paranaense de Energia. Rua Voluntários da Pátria 233, $7^{\circ}$ andar, 80020-942 Curitiba, Paraná, Brasil. 


\section{MATERIAL E MÉTODOS}

Foram analisados 16 exemplares de Siphlophis cervinus (Laurenti, 1768), oito de S. pulcher (Raddi, 1820), quatro de S. longicaudatus, um de S. leucocephalus e um de $S$. worontzowi das seguintes instituições: Field Museum of Natural History, Chicago (FMNH); Instituto Butantan, São Paulo (IB); Museu de História Natural Capão da Imbuia, Curitiba (MHNCI); Field Museum of Natural History, Chicago (FMNH); Museu Paraense Emílio Goeldi, Belém (MPEG); The Natural History Museum, Londres (BMNH); Nationae Naturhistorischen Museum, Leiden (RNHM); e National Museum of Natural History, Washington (USNM).

Para verifícação dos itens alimentares, efetuou-se uma incisão nos escudos ventrais ao nível do estômago e intestino. Os conteúdos foram conservados em álcool $70 \%$ e, juntamente com os exemplares, foram depositados em suas instituições de origem. Foram registrados, para cada exemplar analisado, os itens presentes e a posição pela qual foram ingeridos.

\section{Exemplares analisados}

Siphlophis cervinus. BRASIL, Amazonas: Manaus (IB.9200); Maranhão: Gancho do Ararí (MPEG.14620), Paruá (MPEG. 12835); Pará: Belém (MPEG.170,16468), Capitão Poço (MPEG 6830), Óbidos (IB.14942), Santa Rosa (MPEG. 9323); Rondônia: Porto Velho (IB.40874). BoliviA: Rio Branco (IB.41344). EQUAdOR: Pastaza (USNM.233059). PERU: Moyohamba (BMNH.19294301); Upper Amazon (BMNH.66.2.15.5). TRINIDAD: Caparo (BMNH.192911257). Sem procedência (RMNH.350, FMNH.154508).

Siphlophis pulcher. BRASIL, Rio de Janeiro: São Conrado (IB.53549); Santa Catarina: Florianópolis (IB.17197), Itapoá (MHNCI.334); São Paulo: Caraguatatuba (IB.54225), Santos (IB.10449), Ubatuba(IB.422 19; 55037), Vale do Ribeira (IB.47616).

Siphlophis longicaudatus. Brasil, São Paulo: Campo Limpo (IB.5255), Estação

Evangelista de Souza (IB.10191), São Roque (IB.44214), Tapiraí (IB.28222). Siphlophis leucocephalus. BRAsIL, Goiás: Cana Brava (IB.9141). Siphlophis worontzowi. BRAsIL, Rondônia: Espigão do Oeste (MHNCI.7350).

\section{RESULTADOS E DISCUSSÃO}

Os resultados obtidos encontram-se sumarizados na tabela I. Na amostra analisada de $S$. cervinus foram encontrados restos de lagartos da família Tropiduridae, além de dois novos registros de Scincidae, um de Gymnophthalmidae e um de Gekkonidae. A presença de espécies de Iguanoidea, especialmente de Tropiduridae, apesar de ainda não ter sido registrada na literatura, mostrou-se bastante freqüente ( $27 \%$ dos tratos digestivos analisados) entre os itens alimentares de S. cervinus, em cujo trato digestivo (IB.9200; MPEG.14620) foram encontrados fragmentos de insetos associados a escamas, cauda e patas de Tropiduridae. O exemplar MPEG. 14620 continha restos de Tropiduridae e o exemplar MPEG.16468 continha um espécime de Tropidurus sp.. Uma vez que tanto as espécies de Siphlophis quanto os tropidurídeos amazônicos apresentam hábitos terrestres a semi-arborícolas (NASCIMENTO et al. 1987; SAZIMA \& ARGÔLO 1994; ÁvilA-PIRES 1995), não existe evidências sobre o substrato onde ocorreu a predação. 
Tabela I. Exemplares analisados, localização e posição relativa dos conteúdos do trato digestivo de cada exemplar. Abreviaturas entre parênteses: (e) estômago, (i) intestino, (cd) ingestão iniciada pela região caudal, (ce) ingestão iniciada pela região cefálica.

\begin{tabular}{|c|c|c|}
\hline Espécies & Exemplar & Conteủdo estomacal \\
\hline \multirow[t]{16}{*}{ S. cervinus } & BMNH.66.2.15.5 & Fragmentos de insetos (Formicidae), dentes e escamas de Colubridae (i) \\
\hline & BMNH.1929.4.30.1 & Dentes de serpente (i) \\
\hline & BMNH.1929.11.25.7 & Dentes de serpente (i) \\
\hline & FMNH.154508 & Escamas de Colubridae (i) \\
\hline & IB.9200 & Escamas de Tropiduridae, fragmentos de insetos (Formicidae) (i) \\
\hline & IB. 14942 & Cauda e patas de Mabuya sp., fragmentos de insetos (e, i, ce) \\
\hline & IB. 40874 & Patas de Iguanoidea, fragmentos de insetos (Formicidae) (i) \\
\hline & IB.41344 & Vespertilionidae: Myotis sp. e fragmentos de insetos (e, cd) \\
\hline & MPEG.170 & Patas posteriores de anuro $(e, c e)$ \\
\hline & MPEG. 6830 & Fragmentos de insetos, escamas de lagartos e 1 de serpente (i) \\
\hline & MPEG.9323 & Cauda de Gekkonidae:Gonatodes sp. (e) \\
\hline & MPEG. 12835 & Fragmentos de insetos, ossos e escamas de lagarto (i) \\
\hline & MPEG. 14620 & Fragmentos de Tropiduridae e de insetos (i) \\
\hline & MPEG. 16468 & Tropiduridae (Tropidurus sp.) (e, cd) \\
\hline & RMNH. 350 & Scincidae: Mabuya sp. (e, cd) \\
\hline & USNM.233059 & Fragmentos de Gymnophthalmidae (e, ce) \\
\hline \multirow[t]{8}{*}{ S. pulcher } & IB. 10449 & Dentes e escamas de Mabuya sp. (i) \\
\hline & IB. 17197 & Escamas de Colubroidea (i) \\
\hline & IB.42219 & Escamas de Gymnophthalmidae: Placosoma sp. (i) \\
\hline & IB.47616 & Escamas de lagarto, fragmentos de insetos (i) \\
\hline & IB. 53549 & Escamas de Tropiduridae, fragmentos de insetos (i) \\
\hline & IB. 54225 & $\begin{array}{l}\text { Escamas e fragmentos de patas de Gymnophthalmidae, } 2 \text { escamas de } \\
\text { Tropiduridae (i) }\end{array}$ \\
\hline & IB. 55037 & $\begin{array}{l}\text { Ossos, garras e escamas de lagarto, escamas de Colubroidea e fragmentos } \\
\text { de insetos (i) }\end{array}$ \\
\hline & MHNCI.334 & Anguidae: Ophiodes striatus (e, ce) \\
\hline \multirow[t]{4}{*}{ S. longicaudatus } & IB.5255 & 5 dentes e 2 escamas de Colubroidea (i) \\
\hline & IB. 10191 & $\begin{array}{l}14 \text { dentes e } 2 \text { escamas de Colubroidea; fragmentos de escamas, pele e } \\
\text { ossos de Gymnophthalmidae: Placosoma sp. (i) }\end{array}$ \\
\hline & IB. 28222 & Fragmento cauda e patas posteriores de Polychridae: Enyalius sp. (e, ce) \\
\hline & IB. 44214 & $\begin{array}{l}\text { fragmentos de dedos, escamas e ossos de lagarto; escamas e } 5 \text { dentes de } \\
\text { serpente (i) }\end{array}$ \\
\hline S. leucocephalus & IB.9141 & Fragmentos de dedos de lagarto e insetos (i) \\
\hline S. worontzowi & MHNCI.7350 & Cauda de Gymnophthalmidae: Iphisa elegans (i) \\
\hline
\end{tabular}

Não foi possível identificar a espécie de Gymnophthalmidae encontrada no estômago do exemplar USNM.233059, em função da alteração da região cefálica, decorrente da digestão do exemplar. No entanto, há possibilidade de que pertença a Prionodactylus O'Shaughnessy, 1881 (sensu UzZEL 1973).

No exemplar IB. 14942 foram encontrados fragmentos de insetos, juntamente com cauda e patas de um lagarto do gênero Mabuya Fitzinger, 1826 [possivelmente M. bistriata (Spix, 1825) ou M. nigropunctata (Spix, 1825), segundo a distribuição apresentada por ÁvILA-PIRES (1995)]. Este registro corrobora os dados de alimentação de S. cervinus apresentados por CUNHA \& NASCIMENTO (1993), que citam $M$. bistriata como item alimentar da espécie. O exemplar de $M$. bistriata citado por esses autores corresponde, na verdade, a M. nigropunctata (ÁvILA-PIRES 1995). 
Para o exemplar RMNH.350 não foi possível identificar a espécie de Mabuya encontrada. No entanto, S. cervinus apresenta distribuição amazônica (PETERS \& OREJAS-MiRANDA 1970; CUNHA \& NASCIMENTO 1993), onde também ocorrem, pelo menos, cinco espécies de Mabuya, as quais são encontradas tanto no solo quanto em árvores (até $2,0 \mathrm{~m}$ ), predominantemente em formações abertas (ÁvILAPIRES 1995).

A cauda encontrada no estômago do exemplar MPEG.9323 foi identificada como pertencente a uma espécie de Gonatodes Fitzinger, 1843. Segundo ÁviLA-PIRES (1995), na região de origem do exemplar ocorrem as espécies G. hasemani Griffin, 1917 e G. humeralis (Guichenot, 1855). Este é o segundo registro de uma espécie de Gekkonidae para a dieta de S. cervinus, uma vez que Thecadactylus rapicauda (Houttuyn, 1782) já foi reportado (CUNHA \& NASCIMENTO 1993).

Nos exemplares estudados de $S$. pulcher foram encontrados restos de lagartos das famílias Anguidae, Tropiduridae, Scincidae e Gymnophthalmidae. O registro de Ophiodes striatus (Spix, 1824) corresponde a um novo ítem alimentar para $S$. pulcher e corrobora a afirmativa de que essa espécie forrageia sobre o solo, alimentando-se de vertebrados diurnos (SAZIMA \& ARGÔLo 1994). A região de origem do exemplar MHNCI.334 pode ainda demonstrar que $S$. pulcher habita ambientes inundáveis de restinga higrófila, vegetação predominante local, além do fato de que $O$. striatus é encontrada em abundância neste tipo de ambiente (observação dos autores).

O encontro de escamas de Tropiduridae nos exemplares IB.53549 e IB.54225 sugere que $S$. pulcher habite e forrageie também em áreas de restinga xerofítica. Provavelmente essas escamas sejam de Tropidurus torquatus (Wied, 1820), espécie notadamente com hábitos heliófilos (ZIMMERMAN \& RODRIGUES 1990; JACKSON 1978).

A presença de Scincidae (Mabuya sp.) também constitui-se em um novo registro como ítem alimentar de S. pulcher (IB.10449). Duas espécies de Mabuya, M. caissara Rebouças-Spieker, 1974 e M. macrorhyncha Hoge, 1946 são conhecidas para a costa do Estado de São Paulo, onde habitam moitas de gramíneas e bromélias de chão (REBOUÇAS-SPIEKER 1974). Essa condição complementa a afirmação de que $S$. pulcher alimenta-se sobre o solo.

A ocorrência de fragmentos de Gymnophthalmidae na amostra (IB.54225) e a presença de lagartos do gênero Placosoma Tschudi, 1847 (IB.42219 e O.A.V. Marques, com pess.) corroboram os dados apresentados por SAZIMA \& ARGÔLO (1994) sobre a presença dessa família na dieta de S. pulcher.

Em S. longicaudatus foram encontrados fragmentos de lagarto não identificado (IB.44214), de Placosoma sp. (IB.10991) e de Enyalius sp. (IB.28222). UzZEL (1959) sugeriu que as espécies de Placosoma poderiam ocorrer em árvores. No entanto, as espécies desse gênero ocorrentes na porção paranaense da Floresta Atlântica $[P$. cordylinum Tschudi, 1847 e $P$. glabellum (Peters, 1870)] têm sido normalmente coletadas sobre o solo (observações dos autores e dados da coleção do MHNCI). As espécies de Enyalius Wagler, 1830 do leste do Estado de São Paulo (E. iheringii Boulenger, 1885 e E. perditus Jackson, 1978) ocorrem em áreas da Floresta Atlântica, sendo encontradas desde a superfície do solo até cerca de 1,5 m 
de altura, em arbustos ou troncos de árvores (JACKSON 1978). Isto sugere que $S$. longicaudatus deva forragear sobre o solo. Entretanto, a distribuição vertical dos lagartos utilizados como presa (especialmente Enyalius) evidencia a possibilidade de que esta espécie de serpente possa explorar diferentes locais do ambiente de ocorrência.

Em um exemplar de S. leucocephalus (IB.9141) foram obtidos fragmentos de lagarto não identificado. Já em S. worontzowi (MHNCI.7350) foi encontrada uma cauda de Iphisa elegans Gray, 1851 (Gymnophthalmidae), demonstrando que também essas espécies têm caráter saurófago.

Lagartos em geral são reportados como componentes comuns na alimentação dessas espécies. Dados da literatura atestam a presença, para S. cervinus, das famílias Gekkonidae [Thecadactylus rapicauda (Houttuyn, 1782)], Scincidae[(Mabuya bistriata (Spix, 1825)] e Gymnophthalmidae (Bachia trisanale Cope, 1868) (CUNHA \& NASCIMENTO 1993; DuEllman 1978; NASCIMENTO et al. 1987). Para S. pulcher foram registradas as famílias Gekkonidae (Gymnodactylus Spix, 1825; Hemidactylus Gray, 1817), Gymnophthalmidae (Placosoma Tschudi, 1847) e ovos de lagartos (SAZIMA \& ARGÔLO 1994).

Diversos autores (AMARAL 1933; BEEBE 1946) referiram-se à ofiofagia como um hábito alimentar conhecido para alguns colubrídeos. A única informação sobre predação de serpentes por Siphlophis foi apresentada para S. pulcher, que havia se alimentado de um espécime de Imantodes cenchoa (Linnaeus, 1758) (ver SAZIMA \& ARGÔLO 1994). Neste estudo foram encontradas escamas de serpentes em dois exemplares de S. pulcher (IB.17197 e IB.55037), correspondendo a 25\% dos conteúdos analisados. Em S. cervinus, quatro dos 16 conteúdos analisados (25\%) continham restos de serpentes (dentes e/ou escamas). Também em S. longicaudatus este ítem esteve presente em três dos quatro conteúdos estudados (IB.5255, IB.10191 e IB.44214). A hipótese de que os fragmentos (dentes/escamas) pertençam às próprias serpentes analisadas não pode ser inteiramente descartada. No entanto, especialmente em um conteúdo de S. longicaudatus (IB.10191), esta parece ser uma hipótese remota, dado o grande número de dentes existentes (14). O encontro de escamas e dentes de serpentes nos conteúdos de três espécies de Siphlophis indica que estas podem ser tão importantes na dieta quanto lagartos. Deve-se considerar que a ofiofagia, seja ela preferencial ou não, é registrada em muitas outras espécies dos gêneros da Tribo Pseudoboini (CADLE \& GREENE 1994).

Pequenos mamíferos geralmente fazem parte da dieta alimentar de várias espécies de xenodontíneos sul-americanos. Apesar disso, não existem na literatura registros de predação destes animais por Siphlophis. Neste trabalho registra-se pela primeira vez a predação de quirópteros por um exemplar de S. cervinus (IB.41344). O morcego foi identificado como pertencente ao gênero Myotis Kaup, 1829 (Vespertilionidae), grupo insetívoro (HUSSON 1962). Foi possível observar que a ingestão do animal iniciou-se pela região caudal, não existindo evidências sobre a forma e local da predação.

A falta de dados sobre a anurofagia para esta espécie provavelmente está associada à fácil digestão dos anfíbios. Entretanto, WEHEKIND (1955) inferiu a possibilidade da anurofagia em S. cervinus pelo nome vulgar para a Venezuela e 
Guiana ("yellow frog snake"), mencionado por BEEBE (1946). A presença de restos de anfíbio anuro no trato digestivo do exemplar MPEG.170 de S. cervinus confirma este fato.

CUNHA \& NASCIMENTO (1978) registraram a presença de pássaros na dieta de S. cervinus do leste do Estado do Pará, não indicando o(s) exemplar(es) estudados. Analisando novos exemplares, os mesmos autores não constataram qualquer outro indício de predação de aves por esta espécie de serpente (CUNHA \& NASCIMENTO 1993). A presença de aves na dieta de $S$. cervinus foi também apresentada em DuELLMAN (1989), seguindo CUNHA \& NASCIMENTo (1978). Neste trabalho não se obteve registro de predação de aves por quaisquer das espécies de Siphlophis. A soma das informações disponíveis sugere então, que aves não façam parte da dieta dessas serpentes, o que complementa as suspeitas levantadas por SAZIMA \& ARGÔLO (1994).

Não existem informações fatuais sobre a presença de invertebrados na alimentação de Siphlophis, apesar das informações de AMARAL $(1927,1933)$ sobre a predação de moluscos. No entanto, dada à fácil digestão desses animais e à virtual ausência de vestígios após algum tempo de digestão, esta hipótese não pode ser descartada a priori. A presença de insetos no intestino foi registrada para as espécies S. cervinus, S. pulcher e S. leucocephalus. Segundo MARTINS \& GORDO (1993), o encontro unicamente de artrópodes na porção posterior do intestino sugere uma alimentação à base de anuros. Entretanto, nos casos aqui registrados, a presença de artrópodes está sempre vinculada a vestígios de Squamata (como em seis exemplares de $S$. cervinus e em três de $S$. pulcher). A associação de fragmentos de insetos e de Tropiduridae pode ser explicada pela dieta insetívora apresentada por este grupo de lagartos (ARAÚJO 1991). Por outro lado, exemplares de Formicidae encontrados junto com escamas e dentes de Colubroidea em um exemplar de $S$. cervinus (BMNH.66.2.15.5) podem ter sido ingeridos acidentalmente. Existe também a hipótese de que os restos de insetos encontrados façam parte da dieta da presa do Colubroidea ingerido por $S$. cervinus.

De uma maneira geral, verificou-se a predominância de Squamata dentre os itens alimentares das espécies de Siphlophis. A tabela II sumariza as informações existentes sobre o período de atividade e substrato utilizados pelas espécies de lagartos e serpente predados. Lagartos predominantemente diurnos foram os itens encontrados com maior freqüência. Acredita-se que estes animais possam ter sido predados durante seu período de maior inatividade (à noite), como sugeriram SAZIMA \& ARGÔLO (1994). Apesar do hábito notadamente arborícola manifestado pelas espécies de Siphlophis (DuELLMAN 1989, 1990), a estratégia de caça na superfície do solo parece confirmada pela presença, entre os itens, de espécies fossórias (Bachia trisanale) ou semi-fossórias (Ophiodes striatus), sem hábito escalador.

Hábitos saurófagos e ofiófagos são comuns dentre os Pseudoboini, ocorrendo preferencialmente ou incidentalmente entre as espécies de todos os gêneros relacionados a esta tribo (CADLE \& GREENE 1994). Deve-se considerar que CADLE \& GREENE (1994) sugeriram que anuros e lagartos fizessem parte da dieta primitiva entre os Colubridae neotropicais; poder-se-ia então supor que a predação em lagartos 
seja um caráter plesiomórfico para as espécies do gênero. Um estudo da evolução do tipo de hábito alimentar em Siphlophis é dificultado pela presença de espécies com maior diversidade de itens alimentares, tanto neste gênero (S. cervinus) quanto em outros (por ex., Oxyrhopus Wagler, 1830 e Pseudoboa Schneider, 1801) (CADLE \& GREENE 1994). É importante salientar que a espécie de Siphlophis que apresentou a dieta mais diversificada corresponde àquela com maior número de exemplares analisados, bem como distribuição geográfica mais ampla e, conseqüentemente, com maior possibilidade de diversificação no uso de recursos alimentares segundo os diferentes biomas de sua ocorrência.

Tabela II. Períodos de atividade e substratos de caça dos Squamata encontrados no trato digestivo das espécies de Siphlophis. Abreviaturas e Notações: (+) maior tendência; atividade - (N) noturna, (D) diurna; Substrato - (A) arbóreo, (Ar) arbustivo, (S) sobre o solo, (F) fossória; Registros de literatura: (1) SAZIMA \& ARGólo 1994; (2) CUNHA \& NASCIMENTO 1978; (3) DUELLMAN 1989; (4) Duellman 1990; (5) Zimmerman \& Rodrigues 1990; (6) CunHa \& Nascimento 1993; (7) Ávila-Pires 1995; (8) Duellman 1978; (9) Rodrigues 1988; (10) Uzzel 1973; (11) Rocha 1994; (12) VANZOLINI 1953; (13) MARQUES Comunição pessoal; (14) Observação dos autores, (15) Uzzel 1959; (16) Vanzolini 1948; (17) Vanzolini 1972; (18) RebouçAS-SPIEKER 1974; (19) NASCIMENTO et al. 1987.

\begin{tabular}{|c|c|c|c|c|}
\hline Espéciel Presa & Registros de literatura & Registros originais & Atividade & Substrato \\
\hline \multirow[t]{7}{*}{ S. cervinus } & \multicolumn{2}{|l|}{ Thecadactylus rapicauda $(6,19)$} & $+\mathrm{N}(7)$ & $+A(7)$ \\
\hline & \multicolumn{2}{|l|}{ Bachia trisanale (8) } & $?$ & $F(8)$ \\
\hline & \multirow[t]{5}{*}{ Mabuya nigropunctata $(6,7)$} & & $\mathrm{D}(7)$ & $S, A(7)$ \\
\hline & & Mabuya sp. & $\mathrm{D}(?)$ & $\mathrm{S}, \mathrm{A}(?)$ \\
\hline & & Tropidurus sp. & $D(7,9,14)$ & $\mathrm{S}, \operatorname{Ar}(8,14)$ \\
\hline & & Prionodactylus sp. & $D(7)$ & $S(7,10)$ \\
\hline & & Gonatodes sp. & $D(7)$ & $S, A, \operatorname{Ar}(7)$ \\
\hline \multirow[t]{8}{*}{ S. pulcher } & \multicolumn{2}{|l|}{ Imantodes cenchoa (1) } & $N(2,3,4,5,14)$ & $A, \operatorname{Ar}(3,4,5,14)$ \\
\hline & \multicolumn{2}{|l|}{ Gymnodactylus darwini (1) } & $D(14)$ & $S(1,12)$ \\
\hline & \multicolumn{2}{|l|}{ Hemidactylus mabouia (1) } & $N(7,14)$ & $\mathrm{S}, \operatorname{Ar}(7,14)$ \\
\hline & \multicolumn{2}{|l|}{ Placosoma glabellum (1) } & $D(1,14)$ & $S(13,14), \operatorname{A}-\operatorname{Ar}(14,15)$ \\
\hline & \multirow[t]{4}{*}{ Placosoma sp. (1) } & Placosoma sp. & $D(1,14)$ & $S(13,14), \operatorname{A-Ar}(15)$ \\
\hline & & Ophiodes striatus & $D(14), N(16)$ & $S(16,14)$ \\
\hline & & Tropidurus torquatus & $\mathrm{D}(9,14)$ & $S, \operatorname{Ar}(7,14,17)$ \\
\hline & & Mabuya sp. & $\mathrm{D}(11)$ & $S(18)$ \\
\hline \multirow[t]{2}{*}{ S. longicaudatus } & & Placosoma sp. & $D(1,14)$ & $S(13,14), A-\operatorname{Ar}(14,15)$ \\
\hline & & Enyalius sp. & $D(14)$ & $S(14), \operatorname{A-Ar}(14)$ \\
\hline S. worontzowi & & Iphisa elegans & $\mathrm{N}-\mathrm{D}(7)$ & $S(7)$ \\
\hline
\end{tabular}

AGRADECIMENTOS. A Marcos Di-Bernardo, Márcio Borges Martins, Walter Boeger e Renato Silveira Bérnils pela leitura crítica do manuscrito e diversas sugestões realizadas. A Otávio Augusto Vuolo Marques pelas informações cedidas e sugestões. A Paulo Sérgio Bernarde pelo material coletado. A Juliana Quadros pela identificação do exemplar de Chiroptera. A todos os curadores de museus pelo empréstimo dos exemplares e possibilidade de realização deste trabalho. 


\section{REFERÊNCIAS BIBLIOGRÁFICAS}

AMARAL, A. 1927. Contribuição à biologia dos ophidios brasileiros (habitat, hábitos e alimentação). Col. Trab. Inst. Butantan, São Paulo, 2: 175-181.

- 1933. Mecanismo e gênero de alimentação de serpentes do Brasil. Bol. Biol. (n.s), São Paulo, 1 (1): 2-4

ARAÚJO, A.F.B. 1991. Structure of a white sand-dune lizard community of coastal Brazil. Rev. Brasil. Biol., Rio de Janeiro, 51 (4): 857-865.

ÁvilA-PIRES, T.C. 1995. Lizards of Brazilian Amazonia (Reptilia: Squamata).

Zool. Verh., Leiden, 299: 1-706.

BEEBE, W. 1946. Field notes on the snakes of Kartabo, British Guiana and Caripito, Venezuela. Zoologica, New York, 31 (1): 11-52.

CAdle, J.E. \& H.W. GreEnE. 1994. Phylogenetic patterns, biogeography, and the ecological structure of neotropical snake assemblages, p.281-293. In: R.E. RICKLETS \& D. SCHULTER (Eds). Species diversity in ecological communities: historical and geographical perspectives. Chicago, Univ. Chicago.

CunHA, O.R. \& F.P. NASCIMENTO. 1978. Ofídios da Amazônia. X. As cobras da região leste do Pará. Publ. Avulsos Mus. Par. E. Goeldi, Belém, 31: 1-218.

- 1993. Ofídios da Amazônia. 10. As cobras da região leste do Pará. Bol. Mus. Par. E. Goeldi, ser. zool., Belém, 9 (1): 1-191.

Duellman, W.E. 1978. The biology of an equatorial herpetofauna in Amazonian Ecuador. Misc. Publ. Mus. Nat. Hist. Univ. Kansas, Lawrence, 65: 1-352.

. 1989. Tropical Herpetofaunal communities: patterns of community structure in neotropical rainforests, p.61-88. In: M.L. HARMELIN-VIVIEN \& F. BOURLIÉRE (Eds). Vertebrates in Complex Tropical Systems (Ecological Studies, 69). New York, Springer-Verlag. 1990. Herpetofaunas in neotropical rainforests: comparative composition, history, and resource use, p.455-505. In: A.H. GENTRY (Ed.). Four neotropical rainforests. New Haven, Yale Univ.

Husson, A.M. 1962. The bats of Suriname. Zool. Verh., Leiden, 58: 1-282.

JACKSON, J.F. 1978. Differentiation in the genera Enyalius and Strobilurus (Iguanidae): Implications for Pleistocene climatic changes in Eastern Brazil. Arq. Zool., São Paulo, 30 (1): 01-79.

Martins, M. \& M. Gordo. 1993. Bothrops atrox (Common lancehead). Diet. Herp. Review. 24 (4): 151-152.

Nascimento, F.P., T.C.S. Ávila-Pires \& O.R. CunHa. 1987. Os répteis da área de Carajás, Pará, Brasil (Squamata). II. Bol. Mus. Par. E. Goeldi, ser. zool., Belém, 3 (1): 33-65.

Peters, J.A. \& B. Orejas-Miranda. 1970. Catalogue of the Neotropical Squamata. Part I. Snakes. Bull. U.S. Nat. Mus., Washington D.C., 297 (1): VIII+347.

REBOUÇAS-SPIEKER, R. 1974. Distribution and differentiation of animals along the coast and in continental islands of the State of São Paulo, Brasil. 2. Lizards of the genus Mabuya (Sauria, Scincidae). Papéis Avulsos Zool., São Paulo, 28 (12): 197-240.

RocHA, C.F.D. 1994. Introdução à ecologia de lagartos brasileiros, p.39-57. In: L.B. NASCIMENTO; A.T. BERnARDES \& G.A. COTTA (Eds). Herpetologia no brasil. 1. Belo Horizonte, PUC-MG, Fundação Biodiversitas e Fundação Ezequiel 
Dias.

RoDrigues, M.T. A new anole of the punctatus group from Central Amazonia (Sauria, Iguanidae). Pepéis Avulsos Zool., São Paulo, 36 (29): 333-336.

SAZIMA, I \& A.J.S. ARGÔLO. 1994. Siphlophis pulcher (NCN). Prey. Herp. Review. $25(3): 126$.

Uzzel, T. 1959. Teiid lizards of the genus Placosoma. Occ. Papers Mus. Zool. Univ. Michigan, Ann arbor, 606: 1-16.

1973. A revision of lizards of the genus Prionodactylus, with a new genus

for P. leucostictus and notes on the genus Euspondylus (Sauria, Teiidae).

Postilla, New Haven, 159: 01-67.

VANZOLINI, P.E. 1948. Notas sobre os ofídios e lagartos da Cachoeira das Emas, no município de Pirassununga, Estado de São Paulo. Rev. Brasil. Biol., Rio de Janeiro, 8 (3): 377-400.

1953. Notas sobre alguns lagartos sul-americanos. Rev. Brasil. Biol., Rio de Janeiro, 13 (1): 73-74.

1972. Miscellaneous notes on the ecology of some brazilian lizards (Sauria). Papéis Avulsos Zool., São Paulo, 26 (8): 83-115.

WEHEKIND, L. 1955. Notes on the foods of the Trinidad snakes. British. Jour.

Herpetol., London, 2 (1): 9-13.

Zimmerman, B.L. \& M.T. Rodrigues. 1990. Frogs, snakes, and lizards of the INPA-WWF Reserves near Manaus, Brazil, p.426-454. In: A.H. GENTRY (Ed.).

Four neotropical rainforests. New Haven, Yale University.

Recebido em 27.III.1997; aceito em 15.V.1998. 